\title{
Effect of Damping on the Natural Frequencies of Linear Dynamic Systems
}

\author{
T. K. Caughey and M. E. J. O'Kelly \\ California Instilute of Teclnology, Pasadena, Cabiforniz
}

(Received July 10, 1961)

\begin{abstract}
An analysis is presented of the effect of weak damping onthe natural frequencies of linear dynamic systems. It is shown that the highest natural frequency is always decreased by damping, but the lower natural frequencies may either increase or decrease, depending on the form of the damping matrix.
\end{abstract}

\section{INTRODUCTION}

$I^{N}$ $\mathrm{N}$ his doctoral thesis, Berg ${ }^{1}$ considered the vibration of a dynamic system with generalized linear damping, and showed numerically that the damped natural frequency of the lowest mode was larger than the corresponding frequency of the undamped system. This phenomenon is probably well known to workers in vibration and circuit analysis; however, the authors have been unable to find any systematic treatment of this problem in the literature.

It is well known that in a single-degree-of-freedom system, the damped natural frequency is always less than the undamped natural frequency. In the case of multi-degree-of-freedom systems with classical normal modes, ${ }^{2,3}$ it may be shown that the damped natural frequencies are always less than, or equal to, the corresponding undamped frequencies.

The purpose of this paper is to study the effects of weak damping on the natural frequencies of linear dynamic systems and to show under what conditions the natural frequencies may be increased by damping.

\section{ANALYSIS}

The equations of motion of an $x$-degree-of-freedom linear dynamic system with lumped parameters may be written in matrix notation as

$$
[M]\left\{X^{\prime \prime}\right\}+[C]\left\{X^{\prime}\right\}+[K]\{X\}=\{f(l)\} .
$$

For passive systems the $Y X N^{r}$ matrices $[M]$ and $[K]$ are symmetric and positive definite, and the matrix $[C]$ is symmetric and nonnegative definite.

Consider the homogeneous system obtained by setting $\{f(l)\}=0$ in (1)

$$
[M]\left\{X^{\prime \prime}\right\}+[C]\left\{X^{\prime}\right\}+[K]\{X\}=0 .
$$

\section{CLASSICAL NORMAL MODES}

The system defined by (2) possesses classical normal modes, ${ }^{2}$ if and only if the matrix $[C]$ is diagonalized by the same transformation which simultaneously diagonalizes $[M]$ and $[K]$.

Let

$$
\{X\}=[\Phi]\{\xi\},
$$

${ }^{1}$ G. V. Berg, Ph.D. Thesis, University of Michigan, Ann Arbor, Michigan, 1958

${ }^{2}$ T. K. Caughey, J. Appl. Mech. 27E. 269-271 (1960).

a For the definition of Classical Normal Modes see Appendix. where $[\Phi]$ is the normalized matrix which simultaneously diagonalizes $[M]$ and $[K]$.

If $[C]$ is such that classical normal modes exist, then $[\Phi]^{r}[C][\Phi]=[\bar{C}]$ - a diagonal matrix with elements

$$
\bar{C}_{i}=\left\{\phi^{i}\right\}^{T}[C]\left\{\phi^{i}\right\} .
$$

If (3) is substituted into (2) and then premultiplied by $[\Phi]^{T}$, there results the system of equations:

$$
\bar{M}_{i} \xi_{i}{ }^{\prime \prime}+\bar{C}_{i} \xi_{i}{ }^{\prime}+\bar{K}_{i} \xi_{i}=0, \quad i=1,2, \cdots, \lambda,
$$

where

$$
\begin{aligned}
\bar{M}_{i} & =\left\{\phi^{2}\right\}^{T}[M]\left\{\phi^{i}\right\} \\
\bar{C}_{i} & =\left\{\phi^{i}\right\}^{T}[C]\left\{\phi^{i}\right\} \\
\bar{K}_{i} & =\left\{\phi^{i}\right\}^{T}[K]\left\{\phi^{i}\right\} .
\end{aligned}
$$

Let

$$
\xi_{i}=\xi_{i}^{*} e^{\lambda_{i} t} .
$$

Then

$$
\lambda_{i}=-\frac{\bar{C}_{i}}{2 \bar{M}_{i}} \pm j\left[\frac{\bar{K}_{i}}{\bar{M}_{i}}-\left(\frac{\bar{C}_{i}}{2 \bar{M}_{i}}\right)^{2}\right]^{\frac{1}{2}} \text {. }
$$

Hence, the damped natural frequency is given by:

$$
\omega_{i d}=\left[\omega_{i}{ }^{2}-\left(\frac{\bar{C}_{i}}{2 M M_{i}}\right)^{2}\right]^{\frac{1}{2}}<\omega_{i}, \quad i=1,2, \cdots, x .
$$

Thus, if a system possesses classical normal modes, the damped natural frequencies are always less than, or equal to, the corresponding undamped frequencies.

\section{NONCLASSICAL NORMAL MODES}

If the matrix $[C]$, in (2), is such that it cannot be diagonalized by the transformation which simultaneously diagonalizes $[M]$ and $[K]$, the system is said to possess nonclassical normal modes and must be treated by Foss's method. ${ }^{*}$

To analyze the effect of weak damping on the frequencies in this case, rewrite Eq. (2) in the following manner :

$$
[M]\left\{X^{\prime \prime}\right\}+\epsilon\left[C^{\prime}\right]\left\{X^{\prime}\right\}+[K]\{X\}=0,
$$

where $\epsilon$ is a small parameter. The problem can now be treated by perturbation analysis. ${ }^{5}$

$4 \mathrm{~K}$. A. Foss, "Co-ordinates which uncouple the equations of motion of damped linear systems," Tech. Rept. 25-30. M.I.T. March, 1956.

$\checkmark$ R. Bellman, Inlroduction to Matrix Analysis, (McGraw-Hill Book Company, Inc., New York, 1960). 
Let

$$
\{X\}=\left\{\bar{\phi}^{n}\right\} \exp \bar{\lambda}_{n t}
$$

With substitution of (11) into (10)

Let

$$
\bar{\lambda}_{n}^{2}[M]\left\{\bar{\phi}^{n}\right\}+\epsilon \bar{\lambda}_{n}\left[C^{\prime}\right]\left\{\bar{\phi}^{n}\right\}+[K]\left\{{ }^{n}\right\}=0 .
$$

where $\varphi^{n}$ and $\lambda_{n}$ are the $n$th eigenvector and eigenvalue for the undamped problem, $\epsilon=0$. Inserting (13) and (14) into (12) leads to the following system of equations on separating out the various orders in $\epsilon$ :

$\left(\lambda_{n}^{2}[M]+[K]\right)\left\{\phi^{n}\right\}=0$,

\section{$\left(\lambda_{n}{ }^{2}[M]+[K]\right)\left\{\psi^{n}\right\}$}

$$
=-\left(2[M] \lambda_{n} \mu_{n}+\lambda_{n}\left[C^{\prime}\right]\right)\left\{\phi^{n}\right\}
$$

$$
\begin{aligned}
& \left(\lambda_{n}{ }^{2}[M]+[K]\right)\left\{\theta^{n}\right\} \\
& =-\left[\left(\mu_{n}{ }^{2}+2 \lambda_{n} \nu_{n}\right)[M]+\mu_{n}\left[C^{\prime}\right]\right]\left\{\varphi^{n}\right\} \\
& -\left(2 \lambda_{n} \mu_{n}[M]+\lambda_{n}\left[C^{\prime}\right]\right)\left\{\psi^{n}\right\} .
\end{aligned}
$$

From these equations, the perlurbations of various orders may be calculated.

\section{ZEROTH ORDER SOLUTION}

The zeroth order solution is oblained from Eqs. (15):

$$
\left(\lambda_{n}{ }^{2}[M]+[K]\right)\left\{\varphi^{n}\right\}=0 \quad n=1,2, \cdots, \lambda .
$$

Since $[M]$ and $[K]$ are symmetric and positive definite:

(1) $\lambda_{n}{ }^{2}<0$ all $n$. That is, the eigenvalues $\lambda_{n}$ are pure imaginary.

(2) The $\varphi^{n}$ 's are real.

(3) The $\varphi^{n}$ 's are orthogonal in $[M]$ and $[K]$. That is, $\left\{\varphi^{l}\right\}^{T}[M]\left\{\varphi^{k}\right\}=0 \quad l \neq k$.

In the analysis which follows, it will be assumed for simplicity that the $\lambda_{n}$ 's are distinct.

\section{FIRST-ORDER PERTURBATIONS}

The first-order perturbations are obtained from Eq. (16):

$$
\begin{array}{r}
\left(\lambda_{n}{ }^{2}[M]+[K]\right)\left\{\psi^{n}\right\} \\
=-\left(2[M] \lambda_{n} \mu_{n}+\lambda_{n}\left[C^{\prime}\right]\right)\left\{\varphi^{n}\right\} .
\end{array}
$$

In order to evaluate the first-order perturbations, express $\left\{\psi^{n}\right\}$ in terms of the $\phi^{i}$ 's. Thus

$$
\left\{\psi^{n}\right\}=\sum_{j=1}^{N} a_{n j}\left\{\phi^{j}\right\}
$$

Premultiply Eq. (19) by $\left\{\phi^{l}\right\}^{T}$.

$\therefore \lambda_{n}{ }^{2}\left\{\varphi^{n}\right\}^{T}[M]\left\{\psi^{n}\right\}+\left\{\phi^{2}\right\}^{?}[K]\left\{\psi^{n}\right\}$

$$
=-2 \lambda_{n} \mu_{n}\left\{\phi^{\prime}\right\}^{T}[M]\left\{\phi^{n}\right\}-\lambda_{n}\left\{\phi^{l}\right\}^{T}\left[C^{\prime}\right]\left\{\phi^{n}\right\} \text {. }
$$

Since the $\left\{\phi^{n}\right\}$ may be normalized such that

$$
\left\{\phi^{n}\right\}^{T}[M]\left\{\phi^{n}\right\}=1 \quad n=1,2, \cdots, X .
$$

We have

$$
\left\{\phi^{l}\right\}^{\eta^{\prime}}[M]\left\{\phi^{\prime \prime}\right\}=\delta_{l n}\left\{\phi^{n}\right\}^{T}[M]\left\{\phi^{n}\right\}=\delta_{l n},
$$

where

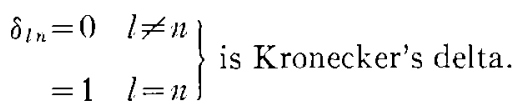

If in Eq. (18), $n$ is replaced by $l$ and the resulting equation transposed, and then post-multiplied by $\left\{\psi^{n}\right\}$ : Then

$$
\lambda i^{2}\left\{\phi^{n}\right\}^{T}[M]\left\{\psi^{n}\right\}+\left\{\phi^{l}\right\}^{T}[K]\left\{\psi^{n}\right\}=0 .
$$

Hence Eq. (21) becomes:

$$
\begin{aligned}
& \left(\lambda_{n}{ }^{2}-\lambda_{l}{ }^{2}\right)\left\{\varphi^{l}\right\}^{T}[M]\left\{\psi^{n}\right\} \\
& =-\left(2 \lambda_{n} \mu_{n} \delta_{1 n}+\lambda_{n}\left\{\varphi^{l}\right\}^{T}\left[C^{\prime}\right]\left\{\varphi^{n}\right\}\right), \\
& \left\{\varphi^{l}\right\}^{T}[M]\left\{\psi^{n}\right\}=-\frac{\lambda_{n}}{\lambda_{n}{ }^{2}-\lambda_{l}{ }^{2}}\left\{\varphi^{l}\right\}^{T}\left[C^{\prime}\right]\left\{\varphi^{n}\right\}
\end{aligned}
$$

If $l=n$, then

$$
\mu_{n}=-\frac{1}{2}\left\{\phi^{n}\right\}^{T}\left[C^{\prime}\right]\left\{\phi^{n}\right\} .
$$

Now premultiply (20) by $\left\{\phi^{l}\right\}^{T}[M]$. Thus

$$
\left\{\phi^{l}\right\}^{T}[M]\left\{\psi^{n}\right\}=\sum_{j=1}^{Y} a_{n j}\left\{\phi^{l}\right\}^{T^{\prime \prime}}[M]\left\{\varphi^{i}\right\}=a_{n l} .
$$

Thus

$$
\begin{aligned}
a_{n l} & =\left\{\phi^{l}\right\}^{\prime \prime}[M]\left\{\psi^{n}\right\}, \\
\therefore a_{n l} & =-\frac{\lambda_{n}}{\lambda_{n l}{ }^{2}-\lambda_{l}{ }^{2}}\left\{\phi^{l}\right\}^{T}\left[C^{\prime}\right]\left\{\phi^{n}\right\}, \quad n \neq l .
\end{aligned}
$$

The quantity $a_{n n}$ is found from the normalization condition

Hence

$$
\left\{\bar{\phi}^{n}\right\}^{T}[M]\left\{\bar{\phi}^{n}\right\}=1 .
$$

$$
a_{n n}=0 .
$$

Therefore, if $\psi^{n}$ can be expanded in terms of $\phi^{j}$,

$$
\psi^{n}=\sum_{j=1}^{W^{\prime}}-\frac{\lambda_{n}}{\lambda_{n}{ }^{2}-\lambda_{j}{ }^{2}}\left(\left\{\phi^{i}\right\}^{T}\left[C^{\prime}\right]\left\{\phi^{n}\right\}\right)\left\{\phi^{i}\right\}
$$

where the symbol $\Sigma^{\prime}$ denotes summation of the indicated values of $j$, omitting the term for which $j=n$.

\section{SECOND-ORDER PERTURBATIONS}

Having determined the first-order perturbations, the second order terms maly be found in a similar manner. Let

$$
\theta^{n}=\sum_{j=1}^{Y} \beta_{n j} \phi^{j} \quad n=1,2, \cdots, \lambda .
$$


With the same technique used above:

$$
\begin{aligned}
\beta_{n l}= & \frac{1}{\lambda_{n}{ }^{2}-\lambda_{l}{ }^{2}}\left[\frac{1}{2}\left(\left\{\varphi^{n}\right\}^{T}\left[C^{\prime}\right]\left\{\varphi^{n}\right\}\right)\left(\left\{\varphi^{l}\right\}^{T}\left[C^{\prime}\right]\left\{\varphi^{n}\right\}\right)\right. \\
& \left.+\sum_{j=1}^{N^{\prime}} \frac{\lambda_{n}{ }^{2}}{\lambda_{n}{ }^{2}-\lambda_{j}^{2}}\left(\left\{\varphi^{j}\right\}^{T}\left[C^{\prime}\right]\left\{\varphi^{n}\right\}\right)\left(\left\{\varphi^{l}\right\}^{T}\left[C^{\prime}\right]\left\{\varphi^{j}\right\}\right)\right] \\
\beta_{n n}= & \left\{\varphi^{n}\right\}^{T}\left[. L I\left\{\theta^{n}\right\}=-\frac{1}{2} \sum_{j=1}^{N} a_{n j}^{2},\right. \\
\nu_{n}= & \frac{1}{8 \lambda_{n}}\left(\left\{\varphi^{n}\right\}^{T}\left[C^{\prime}\right]\left\{\varphi^{n}\right\}\right)^{2} \\
& \quad+\frac{1}{2} \sum_{j=1}^{N} \frac{\lambda_{n}}{\lambda_{n}{ }^{2}-\lambda_{j}^{2}}\left(\left\{\varphi^{j}\right\}^{T}\left[C^{\prime}\right]\left\{\varphi^{n}\right\}\right)^{2}
\end{aligned}
$$

\section{EIGENVECTORS IN DAMPED SYSTEM}

The eigenvectors for the damped system are to terms of order $\epsilon^{2}$ :

$\bar{\phi}^{n}=\varphi^{n}+\epsilon \psi^{n}+\epsilon^{2} \theta^{n}+0\left(\epsilon^{3}\right)$

$$
=\varphi^{n}+\epsilon \sum_{j=1}^{N} a_{n j} \varphi^{j}+\epsilon^{2} \sum_{j=1}^{X} \beta_{n j} \varphi^{j},
$$

where $a_{n j}$ is given by (29) and (30), $\beta_{n j}$ is given by (34) and (35).

\section{Some Interesting Properties of Equation (37)}

(1) If the matrix $\left[C^{\prime}\right]$ is such as to admit classical normal modes, then:

Hence

$$
\left\{\varphi^{i}\right\}^{T}\left[C^{\prime}\right]\left\{\varphi^{\prime \prime}\right\}=0 \quad n \neq j .
$$

$$
\begin{aligned}
& a_{n j}=0 \text { and } \beta_{n j}=0 \quad \begin{array}{l}
j=1,2, \cdots, N \\
n=1,2, \cdots, N^{\prime}
\end{array} \\
& \therefore \bar{\phi}^{n}=\varphi^{n} \quad n=1,2, \cdots, N .
\end{aligned}
$$

That is, the eigenvectors are identical with those for the undamped problem.

(2) If the matrix $\left[C^{\prime}\right]$ is nonclassical, then in general

Now

$$
\left\{\phi^{i}\right\}^{T}\left[C^{\prime}\right]\left\{\varphi^{n}\right\} \neq 0 .
$$

$$
\begin{aligned}
\lambda_{i} & =(-1)^{\frac{3}{3}} \omega_{i} ; \\
\therefore \tilde{\phi}^{n} & =\varphi^{n}+\epsilon(-1)^{\frac{1}{2}} \text { (real vector) } \\
& +\epsilon^{2} \text { (real vector). }
\end{aligned}
$$

Thus, the eigenvectors are, in general, complex.

\section{EIGENVALUES IN DAMPED SYSTEM}

The eigenvalues for the damped system are to terms of order $\epsilon^{2}$ :

$$
\begin{gathered}
\bar{\lambda}_{n}=\lambda_{n}-\frac{\epsilon}{2}\left\{\varphi^{n}\right\}^{T}\left[C^{\prime}\right]\left\{\varphi^{n}\right\}+\epsilon^{2}\left\{\frac{1}{8} \frac{\left(\left\{\varphi^{n}\right\}^{T}\left[C^{\prime}\right]\left\{\varphi^{n}\right\}\right)^{2}}{\lambda_{n}}\right. \\
\left.\quad+\frac{1}{2} \sum_{j=1}^{N^{\prime}} \frac{\lambda_{n}}{\lambda_{n}{ }^{2}-\lambda_{j}^{2}}\left(\left\{\varphi^{i}\right\}^{T}\left[C^{\prime}\right]\left\{\varphi^{n}\right\}\right)^{2}\right\} . \\
\text { Now } \quad \lambda_{n}=(-1)^{\frac{1}{3} \omega_{n} \quad n=1,2, \cdots, N .}
\end{gathered}
$$

Thus

$$
\begin{gathered}
\bar{\lambda}_{n}=(-1)^{1} \omega_{n}\left\{1-\frac{\epsilon^{2}}{2} \sum_{j=1}^{N}\left(\left\{\varphi^{j}\right\}^{T}\left[C^{\prime}\right]\left\{\varphi^{n}\right\}\right)^{2}\left(\omega_{n}^{2}-\omega_{j}^{2}\right)^{-1}\right. \\
\left.-\frac{\epsilon^{2}}{8 \omega_{n}^{2}}\left(\left\{\varphi^{n}\right\}^{T}\left[C^{\prime}\right]\left\{\varphi^{n}\right\}\right)^{2}\right\}-\frac{\epsilon}{2}\left\{\varphi^{n}\right\}^{T}\left[C^{\prime}\right]\left\{\varphi^{n}\right\} \\
\text { DAMPED NATURAL FREQUENCY }
\end{gathered}
$$

\section{DAMPED NATURAL FREQUENCY}

The damped natural frequency for the system is given by ${ }^{6}$

$$
\begin{array}{r}
\omega_{n d}=\omega_{n}\left\{1-\frac{\epsilon^{2}}{2} \sum_{j=1}^{N}\left(\left\{\phi^{j}\right\}^{T}\left[C^{\prime}\right]\left\{\phi^{n}\right\}\right)^{2}\left(\omega_{n}^{2}-\omega_{j}^{2}\right)^{-1}\right. \\
\left.-\frac{\epsilon^{2}}{8 \omega_{n}^{2}}\left(\left\{\varphi^{n}\right\}^{r}\left[C^{\prime}\right]\left\{\varphi^{n}\right\}\right)^{2}+0\left(\epsilon^{4}\right)\right\} .
\end{array}
$$

Some Interesting Properties of Equation (47)

(1) If $\left[C^{\prime}\right]$ is such as to admit classical normal modes then

thus

$$
\left\{\varphi^{j}\right\}^{T}\left[C^{\prime}\right]\left\{\phi^{n}\right\}=0 \quad n \neq j ;
$$

$\omega_{n d l}=\omega_{n}\left\{1-\frac{\epsilon^{2}}{8 \omega_{n}^{2}}\left(\left\{\varphi^{n}\right\}^{T}\left[C^{\prime}\right]\left\{\varphi^{n}\right\}\right)^{2}\right\}$

Hence

$$
n=1,2, \cdots, N \text {. }
$$

$$
\omega_{n d} \leq \omega_{n} .
$$

Equation (49) is in agreement with Eq. (9).

(2) If $\left[C^{\prime}\right]$ is nonclassical, then, in general,

$$
\left\{\phi^{j}\right\}^{T}\left[C^{\prime}\right]\left\{\phi^{n}\right\} \neq 0 \quad n \neq j .
$$

If in (47) $n$ is set equal to $V$,

$$
\begin{gathered}
\omega_{V}>\omega_{N-1}>>\omega_{j}>>\omega_{1}, \\
\therefore \omega_{N^{2}}-\omega_{j}^{2}>0, \\
\therefore \omega_{V d}=\omega_{N}\left\{1-\frac{\epsilon^{2}}{2} \sum_{j=1}^{N^{*}}\left(\left\{\phi^{j}\right\}^{T}\left[C^{\prime}\right]\left\{\phi^{n}\right\}\right)^{2}\left(\omega_{\left.N^{2}-\omega_{j}^{2}\right)^{-1}}\right.\right. \\
\left.-\frac{\epsilon^{2}}{8 \omega_{N}^{2}}\left(\left\{\phi^{n}\right\}^{T}\left[C^{\prime}\right]\left\{\phi^{n}\right\}\right)^{2}\right\} .
\end{gathered}
$$

From Eq. (50), it will be seen that

$$
\text { (3) If } n=1 \text { then } \omega_{N d} \leq \omega_{N} \text {. }
$$

if,

$4 \omega_{1}^{2} \sum_{j=1}^{N^{\prime}}\left(\left\{\phi^{j}\right\}^{T}\left[C^{\prime}\right]\left(\phi^{j}\right\}\right)^{2}\left(\omega_{j}^{2}-\omega_{1}^{2}\right)^{-1}$

$>\left(\left\{\phi^{1}\right\}^{\top}\left[C^{\prime}\right]\left\{\phi^{1}\right\}\right)^{2} . \quad(53)^{7}$

Then $\omega_{1 d}>\omega_{1}$ and Berg's anomalous result is proved.

6 It is of interest to note that the correction in frequency is second order in $\epsilon$, as first pointed out by Rayleigh.

${ }^{7}$ The possibility of satisfying $\mathrm{Eq}$. (53) is increased if the separation between modes is small, i.e, , $\omega_{2} \simeq \widetilde{\omega}_{1}$. 


\section{CONCLUSIONS}

From the above analysis the following conclusions may be drawn:

(1) In a linear dynamic system with weak damping, the damped natural frequency of the highest mode is always less than or equal to the undamped frequency, no matter what form of damping matrix is used.

(2) The damped natural frequency of the lowest mode may be higher than the corresponding undamped frequency, depending on the choice of damping matrix and the mode separation.

(3) In a system with classical normal modes, the damped natural frequencies are always less than or equal to the corresponding undamped frequencies.

Example. To illustrate the results of the above analysis, consider the following system:

$$
[M]\left\{X^{\prime \prime}\right\}+\left[C^{\prime}\right]\left(X^{\prime}\right\}+[K]\{X\}=0,
$$

where

$$
\begin{aligned}
{[M] } & =I \\
{[K] } & =\left[\begin{array}{rrr}
2 & -1 & 0 \\
-1 & 2 & -1 \\
0 & -1 & 2
\end{array}\right] \\
{\left[C^{\prime}\right] } & =\left[\begin{array}{lll}
0 & 0 & 0 \\
0 & 0 & 0 \\
0 & 0 & 1
\end{array}\right] \\
\epsilon & =0.1 .
\end{aligned}
$$

Undamped System. For the undamped system,

$$
\begin{aligned}
& \left\{\phi^{1}\right\}=\frac{1}{2}\left\{\begin{array}{c}
\frac{1}{\sqrt{2}} \\
1
\end{array}\right\}, \quad \omega_{1}=0.765366 ; \\
& \left\{\phi^{2}\right\}=\frac{1}{\sqrt{2}}\left\{\begin{array}{c}
-1 \\
0 \\
1
\end{array}\right\}, \quad \omega_{2}=1.414214 ; \\
& \left\{\phi^{3}\right\}=\frac{1}{2}\left\{\begin{array}{c}
1 \\
-\sqrt{2} \\
1
\end{array}\right\}, \quad \omega_{3}=1.847759 .
\end{aligned}
$$

With use of Eq. (47), the damped natural frequencies are

$$
\begin{aligned}
& \omega_{1 d} \simeq 0.765687>\omega_{1}, \\
& \omega_{2 d} \simeq 1.413993<\omega_{2}, \\
& \omega_{\text {Idd }} \simeq 1.816696<\omega_{3} .
\end{aligned}
$$

The exact values obtained by solving Eq. (54) are

$$
\begin{aligned}
& \omega_{1 d}=0.765688, \\
& \omega_{2 d}=1.413990, \\
& \omega_{3 d}=1.846698 .
\end{aligned}
$$

Comparison of (57) and (58) shows excellent numerical agreement. It should be noted that the damped natural frequency of the first mode is higher than that for the undamped system, while the damped frequencies for the second and third modes are lower than the corresponding values for the undamped system.

\section{APPENDIX}

\section{Classical Normal Modes}

It is well known that undamped linear dynamic systems possess normal modes, and that in each normal mode the various parts of the system pass through their maximum or minimum positions at the same instant of time. Since this type of normal mode was the subject of Lagrange's classic treatise on mechanics, ${ }^{3}$ the author has defined such normal modes as "Classical Normal Modes."

In damped systems in general, it is found that in a normal mode of oscillation, the various parts of the system do not pass through their maximum or minimum position at the same instant of time. In such cases the more general treatment of $\mathrm{F}$. A. Foss ${ }^{4}$ must be used. Rayleigh ${ }^{9}$ showed that if the damping matrix is a linear combination of the stiffness and inertia matrices, the damped system possesses classical normal modes. More recently, Caughey ${ }^{2}$ has shown that a necessary and sufficient condition for the existence of classical normal modes is that the damping matrix be diagonalized by the same transformation which uncouples the undamped system.

${ }^{8}$ J. L. Lagrange, Mechanique Analytique (Gauthier-Villar, Paris, 1811), Nouvelle edition.

${ }^{9}$ Lord Rayleigh, Theory of Sound. (Dover Publications, New York, 1945), Vol. I. 